\title{
Stress components for an infinite plate with a curvilinear hole
}

\author{
F. A. Salama, M. A. Elsayed \\ Department of Mathematics, Faculty of Education, Alexandria University, Egypt \\ Department of basic science High institute for Engineering Elshorouk Academy Egypt
}

\begin{abstract}
In the present paper, the complex variable method is applied to derive exact expressions for Gaursat functions, for the first and second fundamental problems. Here, an infinite elastic plate weakened by a generalized curvilinear hole $C$ is considered, in the two dimensional problems, in the theory of elasticity. The hole is conformally mapped outside the unit circle $\gamma$ by means of a generalized rational mapping function. In addition, when the hole is conformally mapped inside the unit circle, the Gaursat functions are discussed and obtained. Some applications are considered for the first and second fundamental problems. In addition, the components of stress, in each application, are computed using Maple 9.5.
\end{abstract}

Keywords: Gaursat functions, the first and second fundamental problems, conformal mapping, curvilinear hole, stress components.

$\operatorname{MSC}$ (2010): 74B10, 30C20

\section{Introduction}

In the two dimensional problems, in the plane theory of elasticity, it is known that, the first and second fundamental problems are equivalent to finding two analytic functions $\phi_{1}(z)$ and $\psi_{1}(z)$ of one complex argument $z=x+i y$. These analytic potential functions, Gaursat functions, satisfy the boundary conditions

$$
k \varphi_{1}(t)-t \overline{\varphi_{1}^{\prime}(t)}-\overline{\psi_{1}(t)}=f(t),
$$

where $k=-1$ and $f(t)$ is a given function of stress, for the first fundamental problem. While $k=\chi=\frac{\lambda+3 \mu}{\lambda+\mu}>1$, and $f(t)=2 \mu g(t)$ is a given function of the displacement, for the second fundamental problem; $\lambda, \mu$ are called the Lame's constants and $t$ denotes the affix of a point on the boundary. More information for the first and second fundamental problems and its applications can be found in Muskhelishvili [1], Noda and Hetnarski [2], Schinzinger, and Laura [3], and Hetnarski,and Lgnaczak [4]

In terms of the rational mapping function $z=c w(\zeta), c>0, \zeta=\rho e^{i \theta}$; such that $w^{\prime}(\zeta)$ does not vanish or become infinite for $|\zeta|>1$, i.e. the infinite region outside a closed contour conformally mapped outside the unit circle $\gamma$. The two potential functions $\phi_{1}(z), \psi_{1}(z)$, in this case, take the forms

$$
\begin{aligned}
& \varphi_{1}(z)=-\frac{X+i Y}{2 \pi(1+\chi)} \ln \zeta+c \Gamma \zeta+\varphi(\zeta), \\
& \psi_{1}(z)=\frac{\chi(X-i Y)}{2 \pi(1+\chi)} \ln \zeta+c \Gamma^{*} \zeta+\psi(\zeta) .
\end{aligned}
$$

Here, in (1.2) and (1.3) $X, Y$ are the components of the resultant vector of all external forces acting on the boundary and $\Gamma, \Gamma^{*}$ are constants. The two complex functions $\varphi(\zeta)$ and $\psi(\zeta)$ are single valued analytic functions within the region outside the unit circle and $\varphi(\infty)=\psi(\infty)=0$. For the first fundamental problem (stress problem), we have $X=Y=0, \Gamma=\bar{\Gamma}$.

The stress components in the plane theory of elasticity, in the absence of body forces, take the relations, see Muskhelishvili [1].

$$
\begin{gathered}
\sigma_{x x}+\sigma_{y y}=4 \operatorname{Re}\left\{\varphi^{\prime}(z)\right\}, \\
\sigma_{y y}-\sigma_{x x}+2 i \sigma_{x y}=2\left[\bar{z} \varphi^{\prime \prime}(z)+\psi^{\prime}(z)\right] .
\end{gathered}
$$


In this work, the complex variables method will be applied to solve the stress and displacement fundamental problems for an infinite plate weakened by a generalized curvilinear hole $C$ conformally mapped on the domain outside a unit circle $\gamma$ by the generalized rational mapping function

$$
z=c \frac{\zeta+m \zeta^{-\ell}}{1-n \zeta^{-d}}, \quad(|n|<1, z=x+i y, i=\sqrt{-1})
$$

Here, in (1.6), $c>0, m, n,(\ell=1,2, \ldots, P)$ and $d>\ell$ are real parameters restricted such that $w^{\prime}(\zeta) \neq 0, \infty$ outside $\gamma$. The interesting cases when the shape of the hole is a hypotrochoidal a crescent or a cut having the shape of a circular arc are included as special cases. Holes corresponding to certain combinations of the parameters $m, n, \ell, d$ are sketched, see Figs. (1-6). Some applications of the first and second fundamental problems of the infinite plate with a curvilinear hole are investigated and the components of stresses, in each case, are calculating using Maple 9.5.

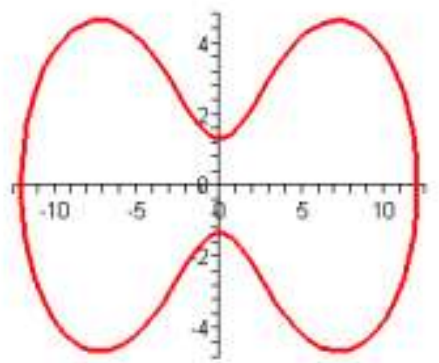

Fig. 1( $c, 2, d, 2, \ell, 1, n, 0.5, m, 2)$

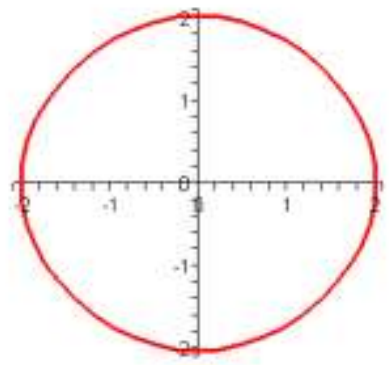

Fig. $3 c, 2, d, 4, \ell, 3, n, 0.01, m, 0.001$

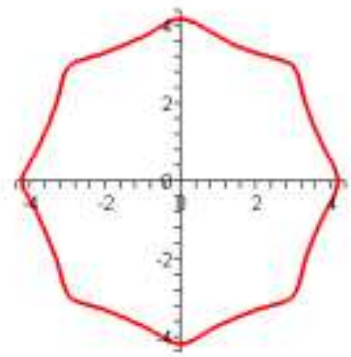

Fig. 5( $c, 4, d, 8, \ell, 5, n, 0.05, m,-0.0001)$

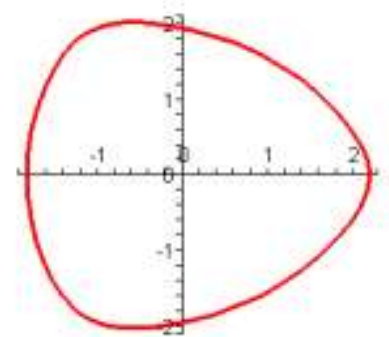

Fig. $2(c, 2, d, 3, \ell, 2, n, 0.1, m,-0.01)$

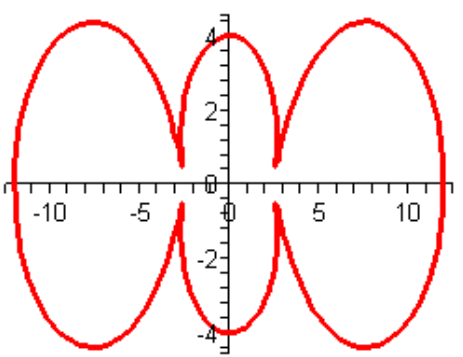

Fig. 4( $c, 2, d, 4, \ell, 1, n, 0.5, m, 2)$

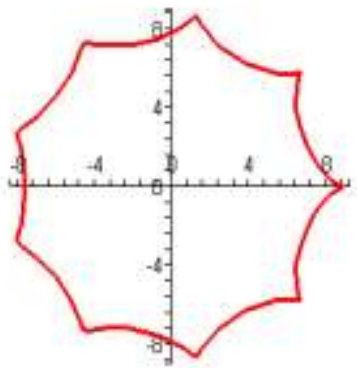

Fig. $6(c, 8, d, 9, \ell, 7, n, 0.075, m, 0.025)$

\section{Gaursat functions}

For determining the Gaursat functions, we write the rational mapping function (1.6) with its first derivatives in the form 


$$
\frac{\omega(\zeta)}{\omega^{\prime}\left(\zeta^{-1}\right)}=\alpha(\zeta)+\overline{\beta(\zeta)}
$$

where

$$
\begin{gathered}
\alpha(\zeta)=\frac{h}{\zeta^{d}-n}, \\
h=\frac{\left(n^{v}+m n^{q}\right)\left(1-n^{2}\right)^{2}}{1-n^{2}(1+d)-l m n^{s}-m p n^{s+2}}, \quad\left\{v=1+\frac{1}{d}, q=1-\frac{l}{d}, s=\frac{1+\ell}{d}\right\}
\end{gathered}
$$

Where, $\beta(\zeta)$ is a regular function for $|\zeta|>1$.

Using (2.1) in the boundary conditions (1.1) and on $\zeta=\sigma$, we get

where

$$
k \phi(\sigma)-\alpha(\sigma) \overline{\phi^{\prime}(\sigma)}-\overline{\psi_{*}(\sigma)}=f_{*}(\sigma),
$$

$$
\begin{aligned}
& \psi_{*}(\zeta)=\psi(\zeta)+\beta(\zeta) \dot{\phi}(\zeta) \\
& f_{*}(\zeta)=F(\zeta)-c k \Gamma \zeta+\frac{c \overline{\Gamma^{*}}}{\zeta}+N(\zeta)[\alpha(\zeta)+\overline{\beta(\zeta)}] \\
& N(\zeta)=c \bar{\Gamma}-\frac{X-i Y}{2 \pi(1+\chi)} \zeta
\end{aligned}
$$

and

$$
F(\zeta)=f(t) .
$$

The function $F(\zeta)$ with its derivatives must satisfy the Hölder condition. Multiplying both sides of (2.4) by $\frac{1}{2 \pi i} \frac{1}{\sigma-\zeta}$ and integrating with respect to $\sigma$ on $\gamma$, we have

$$
-k \phi(\zeta)=A(\zeta)-\frac{c \overline{\Gamma^{*}}}{\zeta}-\frac{h}{\zeta^{d}-n}\left(c b+N\left(n^{v-1}\right)\right),
$$

where

$$
A(\zeta)=-\frac{1}{2 \pi i} \sum_{j=0}^{\infty} \zeta^{-(j+1)} \int_{\gamma} \sigma^{j} F(\sigma) d \sigma,|\zeta|>1 .
$$

and the complex constant $b$, will be determined, is given by

$$
\frac{1}{2 \pi i} \int_{\gamma} \frac{\alpha(\sigma) \overline{\varphi^{\prime}(\sigma)}}{\sigma-\zeta} d \sigma=\frac{c h b}{n^{\nu-1}-\zeta} .
$$

Differentiating (2.6) with respect to $\zeta$, then using the result in (2.8), the complex constant $b$ takes the form

$$
c b=\frac{k E-\eta h \bar{E}}{k^{2}-\eta^{2} h^{2}},
$$

where

$$
E=-\left[\overline{A^{\prime}\left(n^{\nu-1}\right)}+c \Gamma^{*} n^{2(v-1)}+\eta \overline{h N\left(n^{\nu-1}\right)}\right], \quad \eta=\frac{n^{2(v-1)}}{\left(n^{2(v-1)}-1\right)^{2}}
$$

In addition, the function $\psi(\zeta)$ can be determined from (1.1) in the form

$$
\psi(\zeta)=\frac{c k \bar{\Gamma}}{\zeta}-\frac{\omega\left(\zeta^{-1}\right)}{\omega^{\prime}(\zeta)} \phi_{*}(\zeta)+\frac{h \zeta^{d}}{1-n \zeta^{d}} \phi_{*}\left(n^{1-v}\right)+B(\zeta)-B,
$$


where

$$
\phi_{*}(\zeta)=\phi^{\prime}(\zeta)+\overline{N(\zeta)}, \quad B(\zeta)=\frac{1}{2 \pi i} \int_{\gamma} \frac{\overline{F(\sigma)}}{\sigma-\zeta} d \sigma, B=\frac{1}{2 \pi i} \int_{\gamma} \frac{\overline{F(\sigma)}}{\sigma} d \sigma .
$$

The two formulas (2.6) and (2.10) represent the Gaursat functions for the first and second fundamental problems for an infinite elastic plate weakened by a generalized curvilinear hole $C$, that can be mapped outside a unit circle $\gamma$ by the rational mapping (1.6).

An important new case for discussion is using the transformation mapping

$$
z=\frac{\zeta^{-1}+m \zeta^{\ell}}{1-n \zeta^{d}}, \quad|n|<1
$$

This mapping function, when $z^{\prime}(\zeta) \neq 0 ;|\zeta|<1$, transforms the points in the $z$-plane inside the unit circle $\gamma$ in $\zeta$-plane. And, in this case, the Gaursat functions, become

$$
\begin{gathered}
-k \phi(\zeta)=A\left(\zeta^{-1}\right)-c \overline{\Gamma^{*}} \zeta-\frac{h \zeta^{d}}{\left(1-n \zeta^{d}\right)}\left(c b+N\left(n^{\nu-1}\right)\right), \\
\psi(\zeta)=c k \bar{\Gamma} \zeta-\frac{\omega(\zeta)}{\omega^{\prime}\left(\zeta^{-1}\right)} \phi_{*}\left(\zeta^{-1}\right)+\frac{h}{\zeta^{d}-n} \phi_{*}\left(n^{1-v}\right)+B\left(\zeta^{-1}\right)-B .
\end{gathered}
$$

The importance of the conformal mapping that can be transformed inside the unit circle $\gamma$, when $|\zeta|<1$ can be found in Exadaktylos, et al. [5,6].

\section{Special cases}

The importance of the generalized rational mapping (1.6) and the Gaursat functions (2.6) and (2.10) comes from the derived special cases:

(i) For $\ell=1, d=2$, we obtain the same results of El-Sirafy and Abdou [7] and Abdou and Asseri [8], after considering the reality of the coefficients of the mapping function.

(ii) For $\ell=d$, the Gaursat functions (2.6) and (2.10) are agree with the same result of Abdou and Khar-Eldin [9]

(iii) For $n=0$, in (1.6) and for the first fundamental problem the results in agreement with the work of [9] .

(iv) The Gaoursat functions of Eqs. (2.6) and (2.10) when $\ell=1,2, \ldots P$ and $\quad d=\ell+J, J=1,2, \ldots, N$ for the rational mapping of (1.6) can be obtained as new cases in this work.

\section{Applications for Gaursat functions}

1- For $k=-1, \mathrm{X}=\mathrm{Y}=\Gamma=\Gamma^{*}=0$ and $f=-i T t, T$ is a real constant, we have

$$
\begin{aligned}
& \phi(\zeta)=i T c \frac{\left(n^{v}+m n^{q}\right)}{\left(\zeta^{d}-n\right)}-\frac{c h b}{\left(\zeta^{d}-n\right)}, \\
& \psi(\zeta)=-\frac{i T c}{\zeta}-\frac{\omega\left(\zeta^{-1}\right)}{\omega^{\prime}(\zeta)} \varphi^{\prime}(\zeta)+\frac{h \zeta^{d}}{\left(1-n \zeta^{d}\right)} \varphi^{\prime}\left(n^{1-v}\right),
\end{aligned}
$$

where the complex constant $b$ has been determined by Eq. (2.9) and its value was calculated by using Maple 9.5

Thus, (4.1) and (4.2) give the solution of the first fundamental problem for an isotropic infinite plate with a curvilinear hole when there is no external force and the edge of the hole is subject to a uniform tension $(-i T)$.

For a uniform pressure we let in (4.1) and (4.2) $P$ instead of $-i T$.

For $c=2, \ell=1, d=2, m=2, n=0.5$ and $T=0.5$ the relation between the stress components $\sigma_{x x}, \sigma_{y y}, \sigma_{x y}$ and the angle $\theta$, using Maple 9.5 are considered in Figs. $(7,8,9)$. 


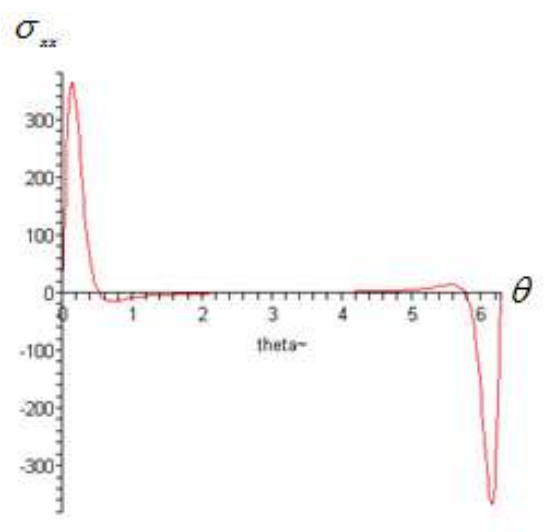

Fig. 7

maximum value of $\sigma_{x x}$ is

$[14.60645,[\theta=5.57807]]$

minimum value of $\sigma_{x x}$ is

$[-14.60645,[\theta=.70512]]$

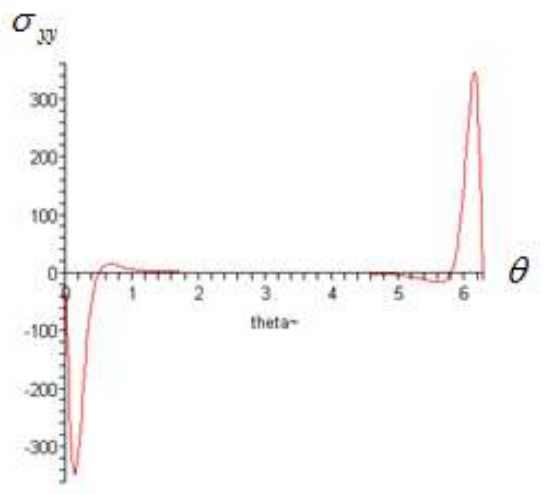

Fig. 8

maximum value of $\sigma_{y y}$ is

$[.18896,[\theta=3.64151]]$

minimum value of $\sigma_{y y}$ is

$[-.18896,[\theta=2.64167]]$

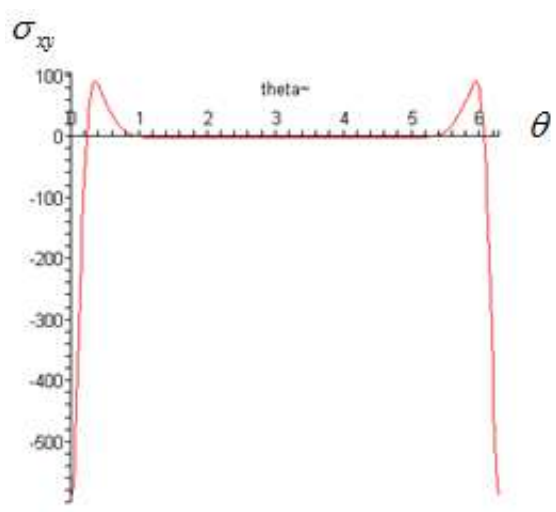

Fig. 9

$$
\begin{aligned}
& \text { maximum value of } \sigma_{x y} \text { is }[90.06610,[\theta=5.93827]] \\
& \text { minimum value of } \sigma_{x y} \text { is }[-2.13908,[\theta=3.38641]]
\end{aligned}
$$

2- For $k=-1, \Gamma=\mathrm{P} / 4, \Gamma^{*}=-\frac{1}{2} P e^{-2 i \vartheta}$ and $\mathrm{X}=\mathrm{Y}=f=0$, we have the Gaursat functions in the form

$$
\begin{aligned}
& \phi(\zeta)=\frac{c P}{2 \zeta} e^{2 i \vartheta}-\frac{h}{\left(\zeta^{d}-n\right)}\left(c b+N\left(n^{\nu-1}\right)\right), \\
& \psi(\zeta)=-\frac{c P}{4 \zeta}-\frac{\omega\left(\zeta^{-1}\right)}{\omega^{\prime}(\zeta)} \phi_{*}(\zeta)+\frac{h \zeta}{\left(1-n \zeta^{d}\right)} \phi_{*}\left(n^{1-\nu}\right),
\end{aligned}
$$

where

$$
\phi_{*}(\zeta)=\phi^{\prime}(\zeta)+\frac{1}{4} c P
$$

The complex constant $b$ has been determined by Eq. (2.9) and its value was calculated by using Maple 9.5 . In this example, we have the infinite plate weakened by the curvilinear hole $C$ which is free from stresses and the plate stretched at infinity by the application of a uniform tensile stress of intensity $P$, making an angle $\vartheta$ with the $x$-axis . 
For $c=2, \ell=1, d=2, m=2, n=0.5, P=1 / 4$ and $\vartheta=\pi / 4$ the relation between the stress components $\sigma_{x x}$ $, \sigma_{y y}, \sigma_{x y}$ and the angle $\theta$ are considered in Figs. $(10,11,12)$

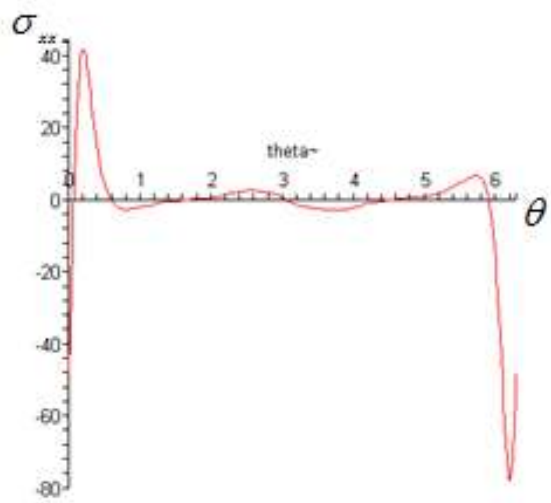

Fig. 10

maximum value of $\sigma_{x x}$ is

$[2.73268,[\theta=2.58786]]$

minimum value of $\sigma_{x x}$ is

$[-2.78151,[\theta=3.67520]]$

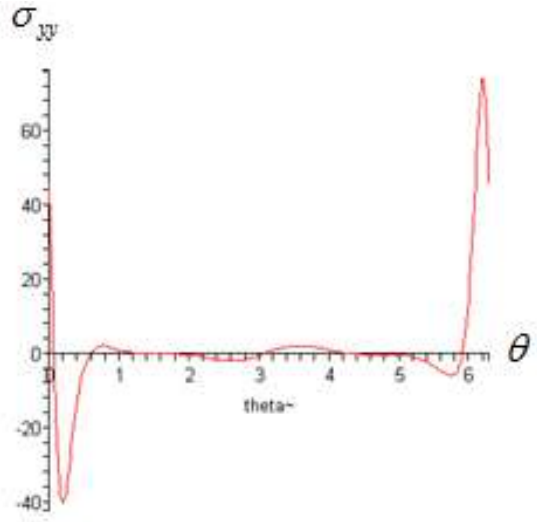

Fig. 11

maximum value of $\sigma_{y y}$ is

[1.93774, $[\theta=3.62569]]$

minimum value of $\sigma_{y y}$ is

$[-1.98863,[\theta=2.62976]]$

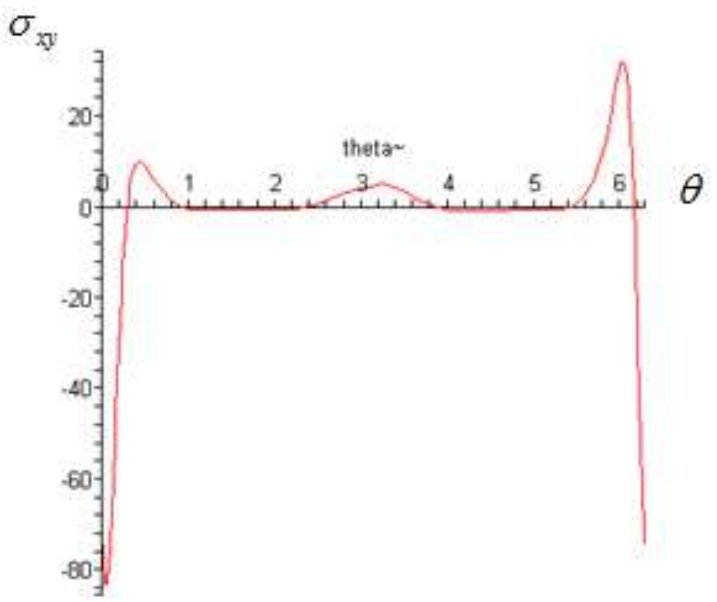

Fig. 12

maximum value of $\sigma_{x y}$ is [5.04813, [ $\left.\left.\theta=3.23076\right]\right]$

minimum value of $\sigma_{x y}$ is [-74.41860, [ $\left.\left.\theta=6.28319\right]\right]$

3- When the external force acts on the center of the curvilinear and the stresses vanish at infinity, i.e. we assume $\Gamma=\Gamma^{*}=f=0, k=\chi$. Here, Goursat functions become

$$
\begin{gathered}
\phi(\zeta)=-\frac{h}{\left(\zeta^{d}-n\right)}\left(c b+N\left(n^{v-1}\right)\right), \\
\psi(\zeta)=-\frac{\omega\left(\zeta^{-1}\right)}{\omega^{\prime}(\zeta)} \phi_{*}(\zeta)+\frac{h \zeta^{d}}{\left(1-n \zeta^{d}\right)} \phi_{*}\left(n^{1-v}\right),
\end{gathered}
$$

where 


$$
\phi_{*}(\zeta)=\phi^{\prime}(\zeta)-\frac{X+i Y}{2 \pi(1+\chi) \zeta}
$$

The complex constant $b$ has been determined by Eq. (2.9) and its value was calculated by using Maple 9.5 . Therefore, we have the solution of the second fundamental problem in the case when a force $X, Y$ acts on the center of the curvilinear kernel.

For $c=2, \ell=1, d=2, m=2, n=0.5, \chi=2$ and $X=Y=10$ the relation between the stress components $\sigma_{x x}, \sigma_{y y}$ ,$\sigma_{x y}$ and the angle $\theta$ are considered in Figs. $(13,14,15)$

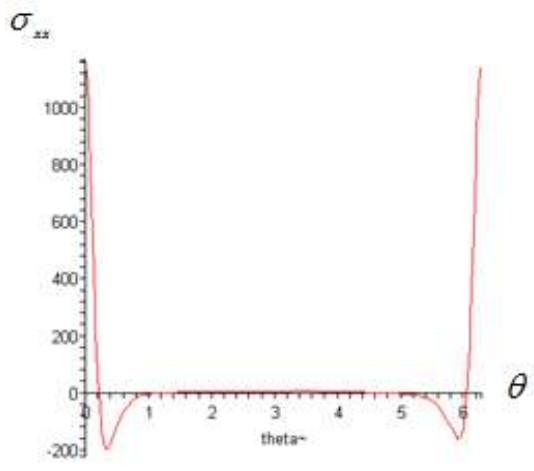

Fig. 13

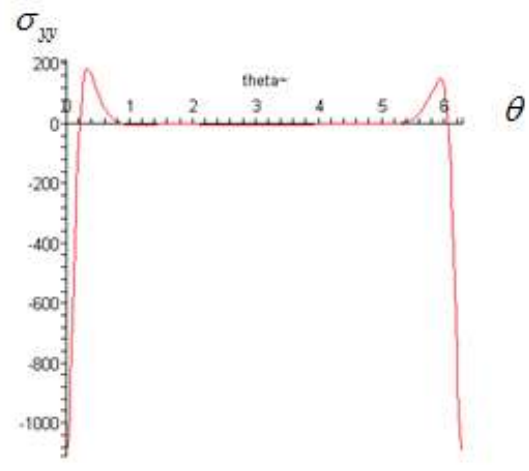

Fig. 14 maximum value of $\sigma_{x x}$ is

$[5.28468,[\theta=2.7461]]$

minimum value of $\sigma_{x x}$ is

$[-160.13870,[\theta=5.91765]]$ maximum value of $\sigma_{y y}$ is

$[184.71993,[\theta=.32408]]$

minimum value of $\sigma_{y y}$ is

$[-5.75047,[\theta=3.40702]]$

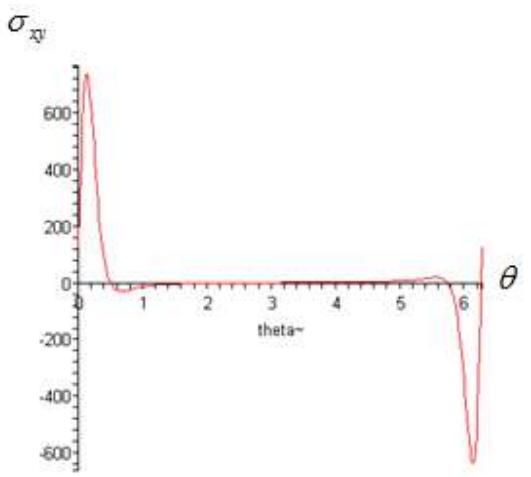

Fig. 15

$$
\begin{gathered}
\text { maximum value of } \left.\left.\sigma_{x y} \text { is [128.72537, [ } \theta=6.28319\right]\right] \\
\text { minimum value of } \sigma_{x y} \text { is }[-29.28010,[\theta=.66047]]
\end{gathered}
$$

\section{Conclusion}

From previous work, the following results can be concluded

1. In the theory of two dimensional linear elasticity one of the most useful techniques for the solution of the boundary value problem for a region weakened by a curvilinear hole is to transform the region into a simpler shape.

2. The mapping (1.6) transforms the domain of the infinite plate with a curvilinear hole in the $z$-plane onto the domain outside a unit circle in $\zeta$-plane under the condition $\omega^{\prime}(\zeta)$ does not vanish or become infinite (when $|\zeta|>1$ ). 
3. The complex variable method ( Cauchy method) is considered one of the best methods for solving the integro-differential equation, boundary value problem, of Eq. (1.1) and obtaining the two complex potential functions, Gaursat functions, $\phi(z)$ and $\psi(z)$ directly.

4. This paper can be considered as a generalization of the work Abdou and Khar-Eldin [9].

\section{References}

[1]. N. I. Muskhelishvili, Some Basic Problems of Mathematical Theory of Elasticity, Noordroof, Holland, 1953

[2]. N. Noda, R. B. Hentarski, Y. Tanigowa, Thermal Stresses, Taylor and Francis, 2003

[3]. R. Schinzinger, P. A. Laura, Conformal Mapping Methods and Applications, Dover Publications, New York, 2003

[4]. R.B. Hetnarski,J. Ignaczak, Mathematical Theory of Elasticity, Taylor and Francis, 2004.

[5]. G. E. Exadaktylos, M. C. Slavropoulou, A closed form elastic solution for stress and displacement around tunnels. Inter. J. of Rock Mech. of Mining sciences 39(2002) 905-916

[6]. G. E. Exadaktylos, P. A. Liolios, M. C. Slavropoulou, A semi-analytical elastic stress-displacement solution for notched circular openings in rocks, Inter. J. of solids and structures 40(2003) 1165-1187

[7]. I. H. El-Sirafy, M. A. Abdou, First and second fundamental problems of infinite plate with a curvilinear hole, J. Math. Phys. Sci. Vol. No.(2) 18 (1984) 205-214

[8]. M. A. Abdou, S. A. Asseri, Gaursat functions for an infinite plate with a generalized curvilinear hole in zeta plane, Appl. Math. Comput. 212(2009) 23-36

[9]. M. A. Abdou,E. A. Khar-Eldin, An infinite plate weakened by a hole having arbitrary shape, J. Comp. Appl. Math. 56(1994) 341351 